\title{
Rapid Diagnostics of a Human Cervical Cancer Cells Under Reflected Light Microscope
}

\author{
Adkham A Paiziev* \\ Institute of Ion-Plasma and Laser Technologies, Academy of Sciences of Uzbekistan, Uzbekistan
}

Submission: January 28, 2019 ; Published: February 07, 2019

*Corresponding author: Adkham A Paiziev, Institute of Ion-Plasma and Laser Technologies, Academy of Sciences of Uzbekistan, Uzbekistan

\begin{abstract}
A technique for revealing surface morphology of human cervical cancer cells has been developed to facilitate early diagnostics of a pre-cancer and cancer cells under reflected light microscopy. The offered method was borrowed from optical microscopy of a solid state surface where the metallographic inverted microscopy (MIM) are usually used. Unlike common accepted transmitted light microscopy for biological applications MIM technique allows to reveal a morphology and topology of a biological cells surface without any treatment by chemicals (fixing, staining, drying, freezing et al). The MIM method was demonstrated by analyzing fresh native smears from epithelium of uterine neck. MIM micrographs of 167 patients with diagnosis cervical cancer allow to visualize on the cancer cells surface numerous of the light reflective formations (LRF). It is supposed that LRF are connected with exocytosis on the cell membrane.
\end{abstract}

Keywords: Cervical cancer; Reflected light microscopy; Cell membrane; Smears

Abbrevations: MIM: Metallographic Inverted Microscopy; LRF: Light Reflective Formations; VIA: Visual Inspection with Acetic Acid; AFM: Atomic Force Microscope

\section{Introduction}

Cervical cancer is the leading cause of cancer death in women population. Most cases of cervical cancer can be prevented through screening action. At present time the key point of accepted in the world screening program is Papanicolau (Pap) test [1]. An abnormal Pap smear is followed by examination of the cervix using a low-power light microscope to visualize changes in tissue reflectance, which might indicate precancer. Implementing this approach requires extensive infrastructure, personnel and economic resources; as a result, the vast majority of women in the world do not have access to life-saving screening programs [2]. Simple visual approaches have been explored to enable cervical cancer screening in resource-poor settings. For example, the use of visual inspection with acetic acid (VIA) is being explored as an alternative to Pap smear screening and colposcopy in many developing countries [2].

Advances in low-cost electronics, have enabled development of sensitive systems for optical imaging and detecting of cervical cancer cells [3] and these optical tools can be used to monitor biologically predictive features of cervical cancer. A number of studies have been carried out to assess whether optical spectroscopy can provide accurate in vivo diagnosis of cervical precancer. Reflectance spectroscopy measures the intensity of light reflected as a function of illumination wavelength, providing information about changes in epithelial cell scattering, stromal scattering and stromal angiogenesis [4].

Currently, optical microscopy techniques are the primary method for cell surface visualization, with microscopic characteristics of cells traditionally used for diagnosis and classification of cancers [1]. However, because the differences in characteristics can be subtle, accurate detection can be challenging and ambiguous [5-6]. Cancer cells are usually identified by their shape and by antibody staining. Usually the nuclei of cancer cells become enlarged, crowded and hyperchromatic.

A technique for permanently capturing a replica impression of biological cells has been developed to facilitate analysis using nanometer resolution imaging tools, namely the atomic force microscope (AFM). The method, termed Bioimprint [7] creates a permanent cell 'footprint' in a polymer composite. The transfer of nanometer scale biological information is presented as an alternative imaging technique at a resolution beyond that of optical microscopy.

The present paper presents an alternative method for studying biological cells using a optical reflected microscope technique, 
to enable imaging of the surface topography of human cervical cancer cells. Here we present a new optical-probe technique based on light-scattering microscopy that is able to detect precancerous and early cancerous changes in cell-rich epithelia.

\section{Methods}

Smears from uterine neck were collected for 167 patients with diagnosis cervical cancer the samples collected were spread on glass slides. Two set of slides were prepared for each patient. The first set of slides were fixed by $95 \%$ ethanol and stained with Papanicolaou dyes in Pathology Department and each slide was then carefully examined by a cytopathologist to distinguish normal cells from leased one. Relevant information was recorded on a specially designed proforma on PC and was marked on the slides. It was clearly specified whether smear was satisfactory or not. Slides showing some abnormal changes in the cellular pattern were further scrutinized by a cytopathologist. Images has been observed on optical microscope "JENAMED-2" and captured by digital high Resolution Microscopy Camera Axio Cam MRc Rev. 3 FireWire. The second set of smears was prepared on glass without fixing any treatment by dyes. This native smears has been observed under optical reflected microscope "Neophot-2". In this case we watched a morphology and topology of cell membrane and captured this images on digital photocamera. This measurement has been performed in Institute of Electronics Uzbek Academy of Science.

\section{Result and Discussion}

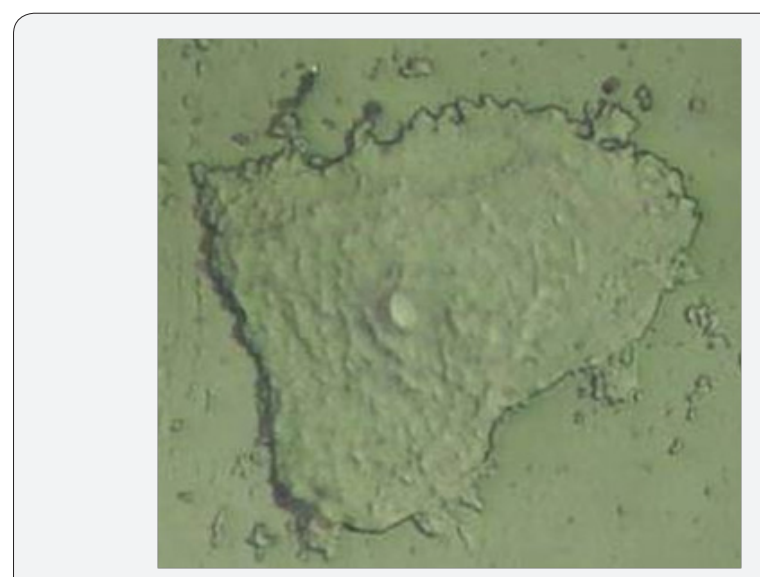

Figure 1: Normal epithelial cells of uterine neck (control smears)

On the Figure 1 we can see image of normal cell surface with pronounced nuclear. But for leased cells so named light reflecting formations (LRF) was observed (Figure 2). The location of the LRF on the cell membrane is shown in Figure 3 too for patients with late stage of disease, where they are seen predominantly concentrated at areas around the nucleus. The diameter of nondysplastic cell nuclei is typically $5 \mathrm{mkm}$, whereas dysplastic nuclei can be as large as $10 \mathrm{mkm}$ and more. Epithelial-cell nuclei can be modeled as transparent spheroids that are large in comparison to the wavelength of visible light (0.4-0.8mm), and whose refractive index is higher than that of the surrounding cytoplasm because of their chromatin content.

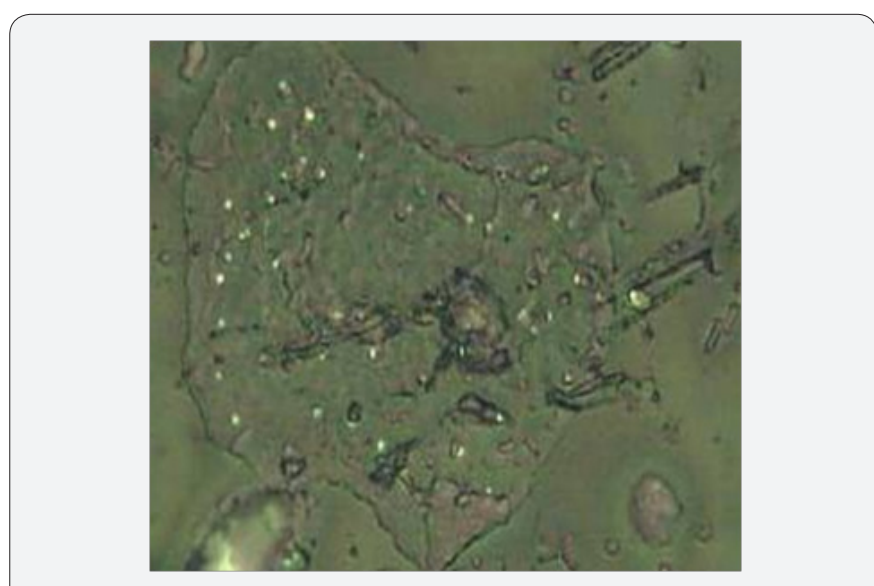

Figure 2: Damaged epithelial cells with diagnosis cervical cancer.

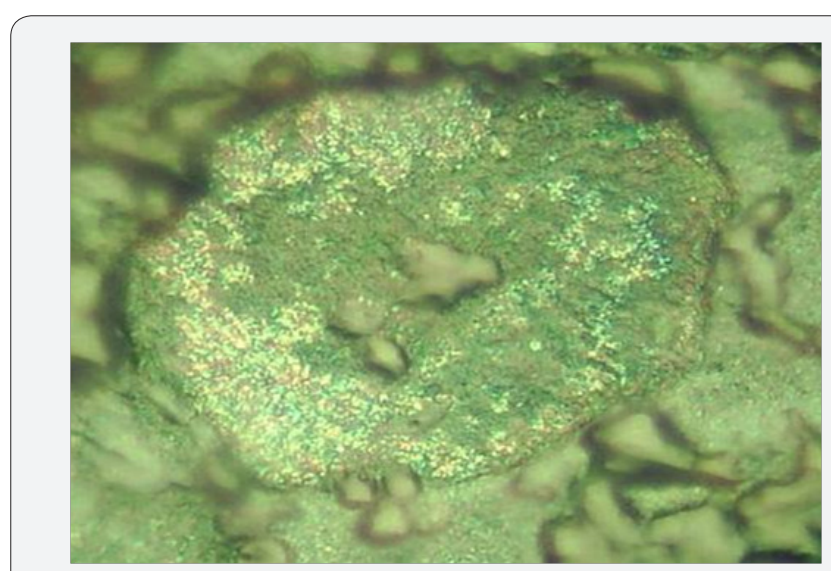

Figure 3: High grade lesion of cervical cancer cells.

For smears of cervix epithelium throughout the field of view of a microscope numerous ballooning-outs, which have a mean size from $0.1-0.5$ to $1.2-1.3 \mathrm{mkm}$, are seen located on the cell surface. The nature and mechanism formation these features of cancer cells is not understood up to now but they are potentially associated with exocytosis. It is accepted that in result of a cancer cell metabolism a granules or vesicles originate inside of cell and move towards cell surface to release its contents. Visualization of such morphological formations has however been limited, partly due to the difficulties with imaging native living or structurally intact cells because convenient transmitted light microscopy technique do not reveal surface cell features which are usually removed after fixing, drying and other treatments of smears.

Much is yet to be known about the nature of cevical cancer cells and until now there has not been a reliable, simple method for cervical cell testing. We suppose that offered method to visualize cell topography in air without fixation and dehydration may be alternative and complementary to Pap-test. Being able to directly view membrane structures regulated by exocytosis will enable researchers to analyze the secretory nature and response of cells, 
yielding insights into drug responses and effects [7]. Considerable variability in the sizes of LRF, as well as dynamic formation and grouping of these structures around the nucleus, illustrates that cells have diverse morphologies.

\section{Acknowledgement}

This work has been supported by Grant № X11-001 of Coordination Center for Science and Technology Republic of Uzbekistan.

\section{References}

1. Koss LG (1989) The Papanicolaou test for cervical cancer detection. A triumph and a tragedy. JAMA 261(5): 737-743.

2. Goldie SJ (2005) Cost-effectiveness of cervical-cancer screening in five developing countries. N Engl J Med 353: 2158-2168.
3. DaCosta RS, Wilson BC, Marcon NE (2007) Fluorescence and spectral imaging. Scientific World Journal 7: 2046-2071.

4. Mirabal YN (2002) Reflectance spectroscopy for in vivo detection of cervical precancer. J Biomed Opt 7(4): 587-594.

5. Hamby L, Sørlie T, Perou CM, Tibshirani R, Aas T, et al. (2002) Gene expression patterns and breast cancer. Cancer Genetics News 4: 1.

6. Palaoro LA, Blanco AM, Gamboni M, Rocher AE, Rotenberg RG, et al. (2007) Usefulness of ploidy, AgNOR and immunocytochemistry for differentiating benign and malignant cells in serous effusions. Cytopathology 18(1): 33-39.

7. Muys JJ, Alkaisi MM, Melville DOS, Nagase J, Sykes P, et al. (2006) Cellular transfer and AFM imaging of cancer cells using Bioimprint. Journal of Nanobiotechnology 4(1): 1-10.

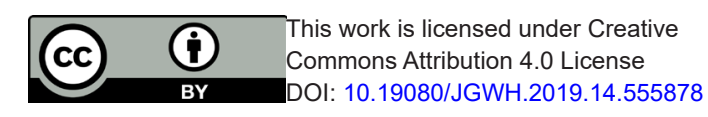


